\title{
ESTABILIDADE E ESTRUTURA DA TURBULÊNCIA SOB A INFLUENCIA DE JATOS DE BAIXOS NÍVEIS NOTURNOS NO SUDOESTE DA AMAZÔNIA
}

\author{
RAONI AQUINO SILVA DA SANTANA ${ }^{1,2}$, JULIO TÓTA ${ }^{1}$, ROSA MARIA NASCIMENTO DOS \\ SANTOS $^{3}$, ROSEILSON SOUZA DO VALE $^{1,2}$
}

\author{
${ }^{1}$ Universidade Federal do Oeste do Pará, Instituto de Engenharia e Geociências, Programa Ciências da \\ Terra, Santarém, PA, Brasil \\ ${ }^{2}$ Instituto Nacional de Pesquisas da Amazônia e Universidade do Estado do Amazonas, Programa de Pós- \\ graduação em Clima e Ambiente, Manaus, AM, Brasil \\ ${ }^{3}$ Universidade do Estado do Amazonas, Escola Superior de tecnologia, Manaus, AM, Brasil
}

raoniass@gmail.com,ieg@ufopa.edu.br,cliamb@inpa.gov.br

Recebido Setembro de 2014 - Aceito Março de 2015

\begin{abstract}
RESUMO
O objetivo deste trabalho foi avaliar como a ocorrência de Jatos de Baixos Níveis (JBNs) noturnos pode influenciar a estrutura da turbulência e a estabilidade atmosférica à superfície. Utilizando-se dados coletados por radiossondagem e pelo sistema de covariância de vórtices turbulentos durante a campanha WetAMC-LBA, foram definidos três regimes de estabilidade atmosférica à superfície: fracamente estável; transição e muito estável. Relacionando estes regimes e a estrutura da turbulência com a ocorrência de JBNs fortes, fracos-tipo 1 (ocorrem acima de $500 \mathrm{~m}$ ) e fracos-tipo 2 (ocorrem até $500 \mathrm{~m}$ ), observou-se que cerca de $22 \%$ dos casos de JBNs fortes estiveram dentro do regime fracamente estável, enquanto que apenas aproximadamente $3 \%$ dos casos de jatos fracos (tipo 1 e tipo 2) encontraram-se neste regime. Outro resultado interessante é que nos casos de JBNs fracos tipo 1, o maior percentual dos pontos encontraram-se dentro do regime muito estável (aproximadamente 54\%). Durante a atividade de JBNs fortes a média da velocidade de fricção e da energia cinética turbulenta foi de $0,09 \mathrm{~ms}^{-1} \mathrm{e} 0,13 \mathrm{~m}^{2} \mathrm{~s}^{-2}$, respectivamente. Para JBNs fracos-tipo 1 estas variáveis apresentaram valores de $0,04 \mathrm{~ms}^{-1} \mathrm{e} 0,02 \mathrm{~m}^{2} \mathrm{~s}^{-2}$, enquanto que para JBNs fracos-tipo 2 os valores atingiram 0,06 $\mathrm{ms}^{-1}$ e $0,03 \mathrm{~m}^{2} \mathrm{~s}^{-2}$. Estes resultados sugerem que os JBNs com velocidades suficientemente altas e dependendo da altura em que ocorrem, podem aumentar a turbulência e introduzir fraca estabilidade atmosférica à superfície.
\end{abstract}

Palavras-chave: Blindagem por cisalhamento; Turbulência; Camada Limite Noturna; Jatos de Baixos Níveis; Amazônia.

\begin{abstract}
STABILITY AND TURBULENCE STRUCTURE UNDER THE INFLUENCE OF THE NOCTURNAL LOW LEVEL JETS IN THE SOUTHWESTERN AMAZON

The aim of this study was to evaluate how the occurrence of nocturnal Low Level Jets (LLJs) may influence the atmospheric turbulence structure and atmospheric stability at the surface. Using data collected from both radiosondes and Eddy Covariance Systems during the WetAMC-LBA campaign, three atmospheric stability regimes were defined at the surface: weakly stable; transition, and very stable. Relating these regimes and the turbulence structure of strong, weak-type 1 (occurring above $500 \mathrm{~m}$ ) and weak-type 2 (occurring below $500 \mathrm{~m}$ ) LLJs, it was observed that $22 \%$ of the strong LLJ cases were within the weakly stable regime, whereas only $3 \%$ of the weak LLJ cases (type 1 and 2) were in this regime. Another interesting result is that in the weak type 1 LLJ cases, the highest percentage were within the very stable regime (approximately 54\%). During strong-LLJs, mean friction velocity and mean turbulence kinetic energy were $0.09 \mathrm{~ms}^{-1}$ and $0.13 \mathrm{~m}^{2} \mathrm{~s}^{-2}$, respectively. For weaktype 1-LLJs, these variables presented respectively values of $0.04 \mathrm{~ms}^{-1}$ and $0.02 \mathrm{~m}^{2} \mathrm{~s}^{-2}$, whereas for weak-type 2-LLJs values were $0.06 \mathrm{~ms}^{-1}$ and $0.03 \mathrm{~m}^{2} \mathrm{~s}^{-2}$, respectively. These results suggest that LLJs
\end{abstract}


with sufficiently high velocities may increase turbulence and introduce weak atmospheric stability at near surface, depending on the height of occurrence.

Keywords: Shear sheltering; Turbulence; Nocturnal Boundary Layer; Nocturnal Low Level Jets; Amazonia.

\section{INTRODUÇÃO}

Fundamentalmente, o conceito de Camada Limite Atmosférica (CLA) está ligado a trocas de energia, massa e momentum que ocorrem entre a superfície terrestre e os primeiros metros da atmosfera (Arya, 2001). O principal mecanismo físico responsável pelo transporte e mistura vertical dessas quantidades nessa camada é a turbulência atmosférica, originada pelo aquecimento do ar em contato com a superfície e pela interação do escoamento atmosférico com os elementos de rugosidade. Contudo, Mahrt (1985), estudando a estrutura vertical da Camada Limite Estável (CLE, ou Camada Limite Noturna, CLN) sob forte estratificação térmica, encontrou como principal fonte de turbulência o cisalhamento do vento próximo ao topo da camada de inversão térmica de superfície. Forte cisalhamento do vento é facilmente verificado durante atuação de Jatos de Baixos Níveis, que, por sua vez, podem provocar aumento da mistura em superfície (Banta et al., 2006; Karipot et al., 2008; Duarte et al., 2012).

Em micrometerologia os Jatos de Baixos Níveis (JBNs) denominam um tipo de perfil vertical de velocidade do vento que apresenta uma forma bem específica, na qual é possível observar um ponto de máximo, também conhecido como núcleo, neste perfil. Tipicamente, os JBNs se propagam numa faixa de altura de 100 a $300 \mathrm{~m}$ acima do solo. Tais jatos são relativamente comuns na CLN, no entanto, ainda está em aberto a discussão sobre como sua ocorrência e intensidade podem afetar a turbulência e a estabilidade atmosférica próxima à superfície. Resultados aparentemente opostos são encontrados, como discutido por Duarte et al. (2012).

Karipot et al, (2008) encontraram que a atividade de JBNs fortes (velocidade do núcleo do jato maior do que 10 $\mathrm{ms}^{-1}$ ) contribuiu para fraca estabilidade atmosférica e alta velocidade de fricção em uma floresta conífera, na Florida. Em contraposição, Prabha et al., (2008) pontuaram que eventos turbulentos de baixa frequência são suprimidos durante a ocorrência de JBNs fortes, sendo isto atribuído ao efeito de "shear sheltering" (traduzido por Nogueira (2008) como: blindagem por cisalhamento).

A teoria de blindagem por cisalhamento foi desenvolvida por Hunt e Durbin (1999) e baseia-se no fato de que vórtices de corrente livre, viajando para uma zona de cisalhamento, podem ser completamente bloqueados, desde que tenham um tamanho e velocidade adequada. Duarte et al., (2012) avaliaram a aplicabilidade desta teoria em Oklahoma, Estados unidos, em um sítio experimental quase ideal, onde não encontraram a presença de blindagem por cisalhamento. Em suas conclusões os autores acreditam que tal teoria não é normalmente aplicada em estudos atmosféricos, uma vez que esta só se aplica em casos bastantes específicos.

Mesmo em regiões extratropicais a questão de como JBNs podem influenciar a estabilidade e a turbulência atmosférica à superfície não está totalmente respondida, fato que se agrava ainda mais quando se trata de regiões tropicais. Na região amazônica poucos trabalhos foram desenvolvidos sobre este tema e se concentram basicamente em descrever o fenômeno de JBNs (Santos, 2005; Santos et al., 2007; Nogueira, 2008).

Baseado nisso, o objetivo deste trabalho foi avaliar a estabilidade e a estrutura da turbulência atmosférica à superfície durante a ocorrência de JBNs de diferentes intensidades. Para avaliar a estabilidade atmosférica primeiramente foram definidos três regimes de estabilidade e em seguida tentou-se identificar se existe preferência de ocorrência de algum regime com a atuação de alguma classe de JBNs.

\section{MATERIAL E MÉTODOS}

A área de estudo compreende uma área desmatada na Amazônia, com terreno relativamente plano, localizada no município de Ouro Preto do Oeste, estado de Rondônia, Brasil ( $10^{\circ} 46^{\prime} \mathrm{S}, 62^{\circ} 20^{\prime} \mathrm{W}$, altitude de $293 \mathrm{~m}$ ). A Fazenda Nossa Senhora (nome pelo qual é conhecida a área de estudo, FNS) é uma área de pastagem estabelecida há aproximadamente 20 anos, coberta por gramínea (Brachiariabrizantha) e que tem sido utilizada em diversos estudos de campo (Figura 1).

De acordo com Fisch (1995), a região onde está localizada a FNS apresenta uma forte sazonalidade na distribuição de precipitação, com totais pluviométricos superiores a $200 \mathrm{~mm} /$ mês durante os meses de novembro a abril (estação chuvosa) e abaixo de $20 \mathrm{~mm} / \mathrm{mês}$ entre os meses de junho a agosto (estação seca). A temperatura do ar também apresenta uma sazonalidade, influenciada pelo regime de precipitação. $\mathrm{O}$ mês mais quente e o mais frio são, respectivamente, outubro $\left(25,5^{\circ} \mathrm{C}\right)$ e julho $\left(22,6^{\circ} \mathrm{C}\right)$.

Os dados utilizados nesta pesquisa foram coletados durante a Campanha de Mesoescala Atmosférica da Estação Úmida (Wet Season Atmospheric Mesoscale Campaign) do experimento LBA - WetAMC-LBA, realizada nos meses de janeiro e fevereiro de 1999. Neste estudo foram utilizados, basicamente, perfis verticais de velocidade do vento, 


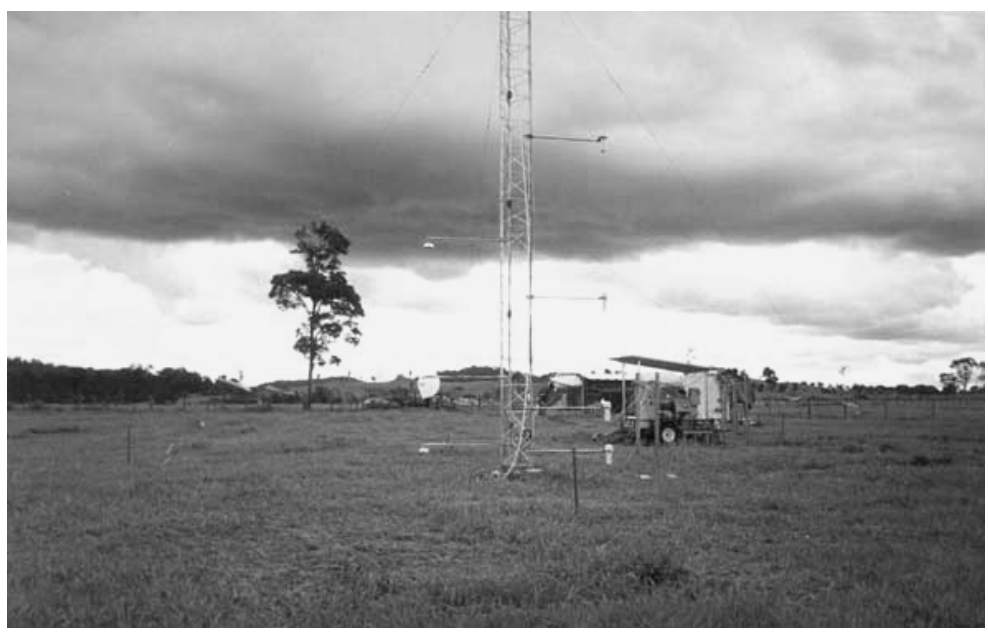

Figura 1 - Vista panorâmica da Fazenda Nossa Senhora, ilustrando a cobertura vegetal e a torre de medidas no centro da imagem.

coletados por radiossondagem atmosférica, e medidas das três componentes do vento (zonal, meridional e vertical), temperatura sônica e umidade, coletadas por um sistema de covariância de vórtices turbulentos. A Tabela 1 sumariza o período de disponibilidade dos dados, taxa de amostragem e equipamentos de medidas utilizados.

Utilizando o sistema "Viz" (Mark II MICROSONDE) a uma taxa de amostragem de $1 \mathrm{~Hz}$, foram lançadas oito radiossondas em intervalos de 3 horas (no período intensivo) e seis radiossondas em intervalos de 4 horas (no período não intensivo) durante o experimento. Aqui apenas perfis entre de 17 horas da tarde até 05 da manhã, hora local (HL), foram utilizados, totalizando um máximo de 5 perfis por noite. Estes dados estão disponíveis em $<$ http://www.lba.iag.usp.br.> para pesquisadores em 4 níveis de controle de qualidade. Neste trabalho foram utilizados os dados da fase 4 , os quais foram submetidos a um controle de qualidade baseado em métodos de inspeção visual, verossimilhança e consistência espacial e física (Longo et al., 2002).

As medidas de covariância de vórtices turbulentos foram realizadas por um anemômetro sônico tridimensional Gill e um analisador infravermelho de gás instalados em uma torre micrometrológica a $6 \mathrm{~m}$ de altura acima do solo
(Veja a Tabela 1 para mais detalhes sobre os instrumentos utilizados). Os dados foram amostrados a uma taxa de $10 \mathrm{~Hz}$ e passaram por inspeção visual para remoção de arquivos que apresentassem inconsistência, devido à falta de energia ou mau funcionamento dos sensores em decorrência de chuva e de outros fatores.

Quanto aos critérios de classificação de JBNs, não há consenso na literatura sobre o assunto, no entanto, muitos dos trabalhos que vem sendo publicados na área utilizam o método de Andreas et al. (2000), propriamente dito, ou com algumas modificações. Estes autores consideraram que JBNs ocorrem se $V_{j}-V_{\text {min }}>2 \mathrm{~ms}^{-1}$, em que $V_{j}$ é a velocidade do núcleo do jato e $\mathrm{V}_{\min }$ a velocidade do vento nos pontos de mínimo, acima e abaixo do núcleo do jato.

Neste trabalho foi utilizado o método de Andreas et al. (2000) para a seleção de JBNs. No entanto considerou-se casos de JBNs quando $\mathrm{V}_{\mathrm{j}}-\mathrm{V}_{\min }>1 \mathrm{~ms}^{-1}$. Além disso, $\mathrm{V}_{\mathrm{j}}$ deve ser maior ou igual a $4 \mathrm{~ms}^{-1} \mathrm{e}$ ocorrer abaixo de $1000 \mathrm{~m}$ de altura acima do solo. Em seguida os JBNs foram classificados de acordo com Karipot et al. (2008), a saber: fracos $\left(4 \mathrm{~ms}^{-1} \leq \mathrm{V}_{\mathrm{j}}<7 \mathrm{~ms}^{-1}\right)$; intermediários $\left(7 \mathrm{~ms}^{-1} \leq \mathrm{V}_{\mathrm{j}}<10 \mathrm{~ms}^{-1}\right)$; e fortes $\left(\mathrm{V}_{\mathrm{j}} \geq 10 \mathrm{~ms}^{-1}\right)$.

A partir dos dados de covariância de vórtices turbulentos foram calculados, a cada 10 minutos, a velocidade de fricção

Tabela 1 - Período de disponibilidade de dados, taxa de amostragem e equipamentos de medidas utilizados.

\begin{tabular}{llll}
\hline & $\begin{array}{c}\text { Período de disponibilidade } \\
\text { de dados }\end{array}$ & $\begin{array}{c}\text { Taxa de } \\
\text { amostragem }\end{array}$ & \multicolumn{1}{c}{ Equipamentos } \\
\hline $\begin{array}{l}\text { Radiossondagem } \\
\text { atmosférica }\end{array}$ & $\begin{array}{l}\text { 09 de janeiro à 28 de } \\
\text { fevereiro de 1999 }\end{array}$ & $1 \mathrm{~Hz}$ & Mark II MICROSONDE \\
\hline $\begin{array}{l}\text { Sistema de } \\
\text { covariância de } \\
\text { vórtices turbulentos }\end{array}$ & $\begin{array}{l}\text { 18 de janeiro a 28 de } \\
\text { fevereiro de 1999 }\end{array}$ & $10 \mathrm{~Hz}$ & $\begin{array}{l}\text { Anemômetro sônico tridimensional } \\
\text { Gill (K12, Gill Instruments Ltd., }\end{array}$ \\
& & & $\begin{array}{l}\text { Lymington, UK) } \\
\text { Analisador infravermelho de gás } \\
\text { (LI-6262, LI-COR inc., Lincoln, } \\
\text { Nebraska, USA) }\end{array}$ \\
\hline
\end{tabular}


(Equação 1), o parâmetro de estabilidade de Monin-Obukhov (Equação 2), a energia cinética turbulenta (Equação 3), o fluxo de calor sensível (Equação 4) e o fluxo de energia cinética turbulenta (Equação 5), de acordo com a seguinte formulação matemática (Stull, 1988):

$$
\begin{aligned}
& u_{*}=\left(\overline{u^{\prime} w^{\prime}}+\overline{v^{\prime} w^{\prime}}\right)^{1 / 4} \\
& \zeta=Z / L \\
& E C T=\frac{1}{2}\left[\overline{\left(u^{\prime}\right)^{2}}+\overline{\left(v^{\prime}\right)^{2}}+\overline{\left(w^{\prime}\right)^{2}}\right] \\
& H=\rho c_{p} \overline{\theta^{\prime} w^{\prime}} \\
& F_{E C T}=\overline{E C T w^{\prime}}
\end{aligned}
$$

Sendo: $u$ ' e $v$ ' as partes turbulentas do vento zonal e meridional, respectivamente; $\mathrm{w}$ ' a parte turbulenta da velocidade vertical; $\mathrm{Z}$ a altura; $\rho$ a densidade do ar seco; $c_{p}$ o calor específico a pressão constante; $\theta$ ' a parte turbulenta da temperatura potencial; $L \mathrm{o}$ comprimento de Obukohv (Equação 6), dado por:

$$
L=-\frac{u_{*}^{3}}{k \frac{g}{T_{0}} \overline{\theta^{\prime} w^{\prime}}}
$$

em que: $T_{0}$ é a temperatura média do ar na altura $z ; k$ é a constante de Von Kármán;

Para definir regimes de estabilidade atmosférica, seguiu-se a metodologia proposta por Mahrt et al. (1998), que relaciona $\zeta$ e o fluxo cinemático de calor (aqui este fluxo foi convertido para fluxo de calor sensível ${\mathrm{em} \mathrm{Wm}^{-2}}^{-2}$. Estes autores definiram a ocorrência de três regimes de estabilidade para a Camada Limite Estável: fracamente estável $\left(0<\zeta<\varepsilon_{1} 1\right)$, o qual é caracterizado pelo aumento do fluxo de calor a medida que $\zeta$ aumenta; transição $\left(\varepsilon_{1}<\zeta<\varepsilon_{2}\right)$, onde o fluxo de calor decai rapidamente com o aumento de $\zeta$; e muito estável, no qual o fluxo de calor é muito pequeno e continua a decair lentamente com o aumento da estabilidade ( $\varepsilon_{1}$ e $\varepsilon_{2}$ são limiares que separam cada regime de estabilidade, mais detalhes na seção 3.2).

\section{RESULTADOS E DISCUSSÃO}

\subsection{Jatos de Baixos Níveis}

Os JBNs tiveram presença constante nas noites aqui estudadas (veja a Tabela 2). Cerca de $60 \%$ dos perfis de vento analisados apresentaram tais características, ou seja, 142 perfis, de um universo de 235 , foram considerados como JBNs, sendo que destes 76 foram classificados como casos fracos (53\%),
45 intermediários (32\%) e 21 fortes (15\%). O percentual de ocorrência encontrado aqui é bem maior do que o apresentado na grande maioria dos trabalhos realizados da Amazônia (Santos, 2005, Nogueira, 2008). Isto se deve principalmente devido a diferenças nos critérios de seleção de jatos utilizados.

A Figura 2 mostra alguns perfis de vento que apresentaram JBNs. São exemplos de JBNs fracos os perfis das Figuras $2 \mathrm{~A}$ e 2B, com $\mathrm{V}_{\mathrm{j}}$ de $4,2 \mathrm{~ms}^{-1}$ e $4,1 \mathrm{~ms}^{-1}$ e $\mathrm{Z}_{\mathrm{j}}$ de 500 $\mathrm{m}$ e $200 \mathrm{~m}$. Os JBNs intermediários estão representados nos perfis das Figuras 2F, 2G, 2H e 2I, com $\mathrm{Z}_{\mathrm{j}}$ entre $220 \mathrm{~m}$ e $660 \mathrm{~m}$. Os perfis de vento das Figuras 2C, 2D, e 2E exemplificam jatos fortes, os quais apresentam características bem distintas, enquanto nos dois primeiros $Z_{j}$ está abaixo de $500 \mathrm{~m}$, no último $Z_{j}$ ocorre em $780 \mathrm{~m}$ e apresenta $V_{j}$ de $16,4 \mathrm{~m} / \mathrm{s}$. É importante notar também a presença de mais de um máximo em muitos dos perfis amostrados, ficando mais evidente nas Figuras 2C, $2 \mathrm{D}, 2 \mathrm{~F}$ e $2 \mathrm{H}$.

Apesar de JBNs de diferentes intensidades poderem ocorrer, tanto mais próximo da superfície, quanto no limite superior de altura estabelecido (1000 m de altura acima do solo), nossos resultados mostram que jatos fortes tendem a acorrer em maiores alturas (Figura 3). Este resultado já era esperado, uma vez que o atrito do ar com a superfície desacelera o escoamento. Por outro lado, jatos fracos se distribuem por todas as altitudes (Figura 3). É importante salientar, porém, que se olhou para a média de $Z_{j}$ para os três diferentes tipos de JBNs (Tabela 2) e percebeu-se que ela aumenta à medida que a média de $\mathrm{V}_{\mathrm{j}}$ aumenta.

Na Amazônia JBNs vêm sendo documentados desde 1992 (Greco et al., 1992), sempre tentando entender qual seria o mecanismo responsável pela formação do fenômeno. Oliveira e Fitzjarrald (1993) identificaram o fenômeno de brisa de rio em uma área de floresta próximo a cidade de Manaus, sendo este um possível mecanismo para a formação de JBNs. Cohen et al., (2006) mostraram que JBNs que ocorreram a leste do Pará, resultam de um fenômeno de "canalização" do escoamento a nordeste do estado do Pará, havendo intensificação do vento quando a direção deste segue aquela dos grandes cursos d'água.

Por se tratar de uma área longe da influência de rios e de efeitos orográficos, conjeturou-se que os JBNs mais baixos encontrados neste trabalho aparecem provavelmente devido ao desacoplamento friccional do ar acima da inversão térmica, o chamado efeito de Blackadar (Blackadar, 1957). Já em relação

Tabela 2 - Estatística dos JBNs.

\begin{tabular}{lcccccc} 
& $\begin{array}{c}\mathrm{V}_{\mathrm{j}}\left(\mathrm{ms}^{-1}\right) \\
\text { Min-Max }\end{array}$ & $\begin{array}{c}\mathrm{V}_{\mathrm{j}} \text { médio } \\
\left(\mathrm{ms}^{-1}\right)\end{array}$ & $\begin{array}{c}\mathrm{Z}_{\mathrm{j}}(\mathrm{m}) \\
\text { Min- } \\
\text { Max }\end{array}$ & $\begin{array}{c}\mathrm{Z}_{\mathrm{j}} \text { médio } \\
(\mathrm{m})\end{array}$ & $\begin{array}{c}\text { Número } \\
\text { de perfis }\end{array}$ & $\begin{array}{c}\text { Percentual de } \\
\text { ocorrência }\end{array}$ \\
\hline JBNs fracos & $4,1-6,9$ & 5,6 & $140-950$ & 477 & 76 & 53 \\
JBNs intermediários & $7,1-9,9$ & 8,2 & $160-740$ & 525 & 45 & 32 \\
JBNs fortes & $10,1-18,3$ & 12,1 & $360-940$ & 677 & 21 & 15
\end{tabular}





Figura 2 - Amostragem de perfis de velocidade do vento obtidos a partir de dados de radiossondagem atmosférica, exemplificando casos de JBNs de diferentes intensidades. As setas indicam as coordenadas do núcleo do jato.



Figura 3 - Velocidade do núcleo do jato em relação à altura do núcleo do jato, para todos os casos de JBNs identificados na FNS.

aos JBNs que se propagam em maiores altitudes, efeitos de meso e grande escala, por si só, ou combinado com o efeito de Blackadar, podem ser responsáveis pelo aparecimento dessa categoria de jato. Tal resultado está em consonância com o encontrado por Greco et al., (1992) e Santos (2005).

\subsection{Regimes de estabilidade}

Com intuito de verificar a relação entre a estabilidade atmosférica à superfície e a ocorrência de JBNs fortes e fracos, primeiramente definiu-se regimes de estabilidade para a área de estudo. Foram utilizados 2182 períodos de 10 minutos fornecidos pelo sistema de covariância de vórtices turbulentos das $18 \mathrm{HL}$ às $06 \mathrm{HL}$, remanescentes depois de excluídos os períodos de 10 minutos com $\mathrm{H}$ positivo e $\zeta>10$, como em Mahrt et al. (1998). Para se obter os limiares $\varepsilon_{1}$ e $\varepsilon_{2}$, foi feita a média de $\zeta$ e H para os seguintes intervalos de $\zeta:[0,0010,005[;[0,0050,01[;[0,010,05[$; [0,05 0,1[; [0,1 0,5[; [0,5 1[; [1 5[; [5 10]. Aqui, como em Mahrt et al. (1998), três regimes foram definidos (Figura 4), a saber: fracamente estável $(\zeta \leq 0,03)$; transição $(0,03<\zeta \leq 0,71)$ e muito estável $(\zeta>0,71)$. Os limiares $\varepsilon_{1}=0,03$ e $\varepsilon_{2}=0,71$ podem variar dependo do período de tempo utilizado no cálculo de $\mathrm{H}$ e $\zeta$, ou dos intervalos escolhidos no cálculo da média destas variáveis.

No regime fracamente estável a magnitude do fluxo de calor sensível aumenta com o aumento $\zeta$. Segundo Mahrt et al. (1998) e Mahrt (1999), isso acontece devido às amplificações nas flutuações da temperatura. Analisando o conjunto de dados, verificou-se que a partir de $\zeta=0,03$, o fluxo cinemático de calor decaiu. Inicialmente essa queda foi relativamente rápida, o que caracteriza o regime de transição, no entanto, quando $\zeta$ atingiu um valor igual a, o decaimento foi mais suavizado e o fluxo de calor muito pequeno quando comparado com seu máximo, uma vez que a estabilidade restringe a turbulência, assim diz-se que a CLN está em um regime de estabilidade atmosférica muito estável.

\subsection{Estabilidade e turbulência sob a influência de JBNs}

Karipot et al. (2008), estudando em floresta conífera na Florida, Estados Unidos, e utilizando o número de Richardson (Ri) como parâmetro de estabilidade, mostraram que a presença de JBNs fortes introduzem fraca estabilidade e $u_{*}$ alto à 


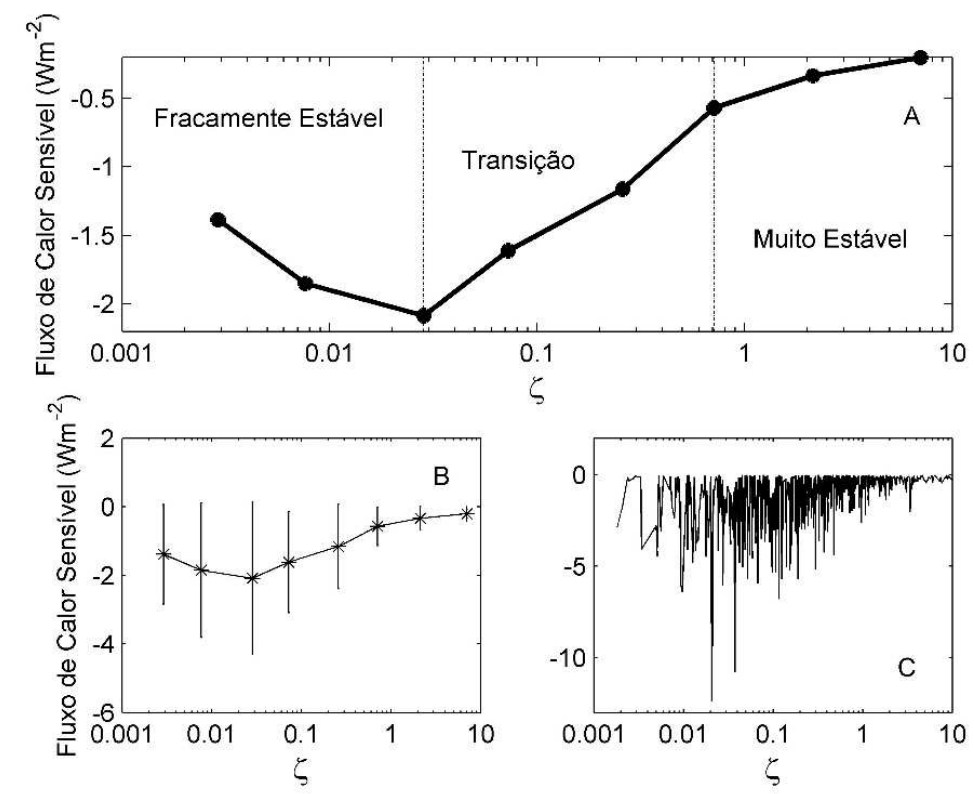

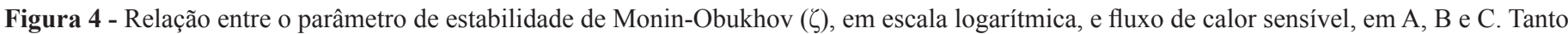
em A quanto em B, os pontos representam médias para diferentes intervalos de $\zeta$ (tais intervalos estão especificados no texto). Em B as barras de erro representam o desvio padrão de cada ponto para o fluxo de calor sensível. No quadro A, as Linhas verticais tracejadas indicam limiares para cada regime de estabilidade. No quadro C estão representados todos os pontos utilizados no cálculo das médias.

superfície, enquanto que JBNs fracos resultam em condições de estabilidade atmosférica de moderada a muito estáveis, além de fraca turbulência atmosférica à superfície. Aqui um estudo similar foi realizado, mas utilizando os regimes de estabilidade supracitados, definidos pelo parâmetro de estabilidade de Monin-Obukhov.

Para fazer está análise calculou-se H, ECT, u*, fluxo de ECT e $\zeta$ à superfície durante a hora correspondente aos eventos de JBNs fortes, fracos-tipo $1(\mathrm{Zj}>500)$ e fracos-tipo $2(\mathrm{Zj} \leq 500)$. Uma vez que cada variável é calculada a cada 10 minutos, cada evento de jato pode apresentar até seis pontos correspondentes à superfície. Como na seção anterior, também foram excluídos os períodos com $\mathrm{H}$ positivo e $\zeta>10$. Infelizmente, devido falta de dados de turbulência não foi possível analisar todos os casos de JBNs identificados neste trabalho.

Comparando os casos de JBNs fortes com os de JBNs fracos-tipo 1 , nota-se que em média maior turbulência atmosférica é observada à superfície. A média de $u_{*}$ é mais que dobro maior durante eventos de JBNs fortes em relação aos de JBNs fracos-tipo 1. A mesma tendência é mostrada pela ECT, no entanto esta é cerca de seis vezes maior (Tabela 3 ). A Figura 5 mostra a dispersão dos pontos para as estas duas classes de JBNs. Para o fluxo de ECT os pontos estiveram muito próximos de zero ou positivos nos dois casos, exceto dois pontos (triângulos pretos dentro das elipses, Figura 5D), em que atuação de um jato forte, viajando a uma altura de 320 $\mathrm{m}$, provavelmente transportou para a superfície turbulência gerada em níveis superiores da atmosfera (Mahrt,1999; Banta et. al., 2002; Karipot et. al., 2008; Duarte et. al., 2012). Neste caso, pode-se dizer que existiu uma camada limite invertida, da maneira como definida por Mahrt (1999).

As diferenças entre JBNs fortes e JBNs fracos-tipo 2 são menos pronunciadas (Figura 6), ainda que maiores valores médios de $u_{*}$ e ECT sejam observados durante eventos de JBNs

Tabela 3 - Pontos, variáveis turbulentas (o operador “<>” representa as médias dessas variáveis) e número de casos dos regimes de estabilidade para três classes de JBNs.

\begin{tabular}{ccccccccc}
\hline & $\begin{array}{c}\mathrm{N}^{\circ} \text { de } \\
\text { perfis }\end{array}$ & $\begin{array}{c}\mathrm{N}^{\circ} \text { de } \\
\text { pontos em } \\
\text { superfície } \\
(10 \text { min })\end{array}$ & $\begin{array}{c}\langle\boldsymbol{H}\rangle \\
\left(\mathrm{Wm}^{-2}\right)\end{array}$ & $\begin{array}{c}\langle\boldsymbol{E C T}\rangle \\
\left(\mathrm{m}^{2} \mathrm{~s}^{-2}\right)\end{array}$ & $\begin{array}{c}\left\langle\boldsymbol{u}_{*}\right\rangle \\
\left(\mathrm{ms}^{-1}\right)\end{array}$ & $\begin{array}{c}\text { Fracamente } \\
\text { estável }\end{array}$ & $\begin{array}{c}\text { Inter } \\
\text { medi } \\
\text { ário }\end{array}$ & $\begin{array}{c}\text { Muito } \\
\text { estável }\end{array}$ \\
\hline $\begin{array}{c}\text { JBNs fracos-tipo 1 } \\
(\mathrm{Zj}>500)\end{array}$ & 9 & 37 & $-0,6$ & 0,02 & 0,04 & 1 & 16 & 20 \\
\hline $\begin{array}{c}\text { JBNs fracos-tipo } 2 \\
(\mathrm{Zj} \leq 500)\end{array}$ & 18 & 87 & $-1,1$ & 0,03 & 0,06 & 3 & 57 & 27 \\
\hline JBNs fortes & 6 & 32 & $-1,2$ & 0,13 & 0,09 & 7 & 17 & 8 \\
\hline
\end{tabular}



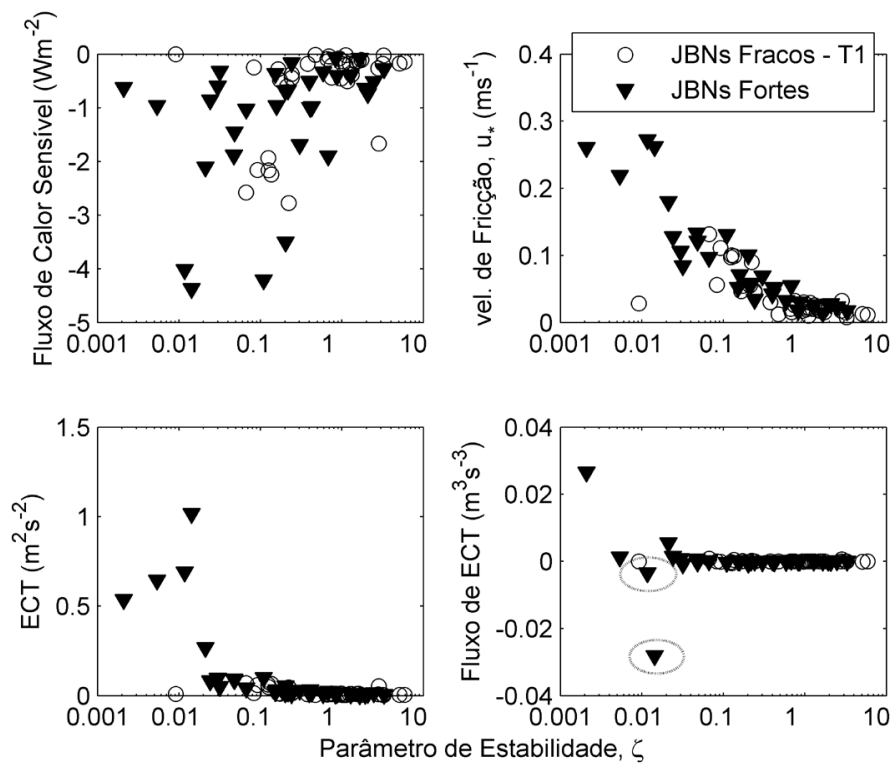

Figura 5 - Dispersão do parâmetro de estabilidade Monin-Obukhov em relação ao fluxo de calor sensível (A), velocidade de fricção (B), energia cinética turbulenta (C) e fluxo de energia cinética turbulenta (D), calculados durante eventos de JBNs fortes (triângulos pretos) e fracos-tipo 1 (círculos brancos). Os pontos dentro das elipses são correspondentes a um evento de jato forte que apresentou $\mathrm{Z}_{\mathrm{j}}$ de $320 \mathrm{~m}$.
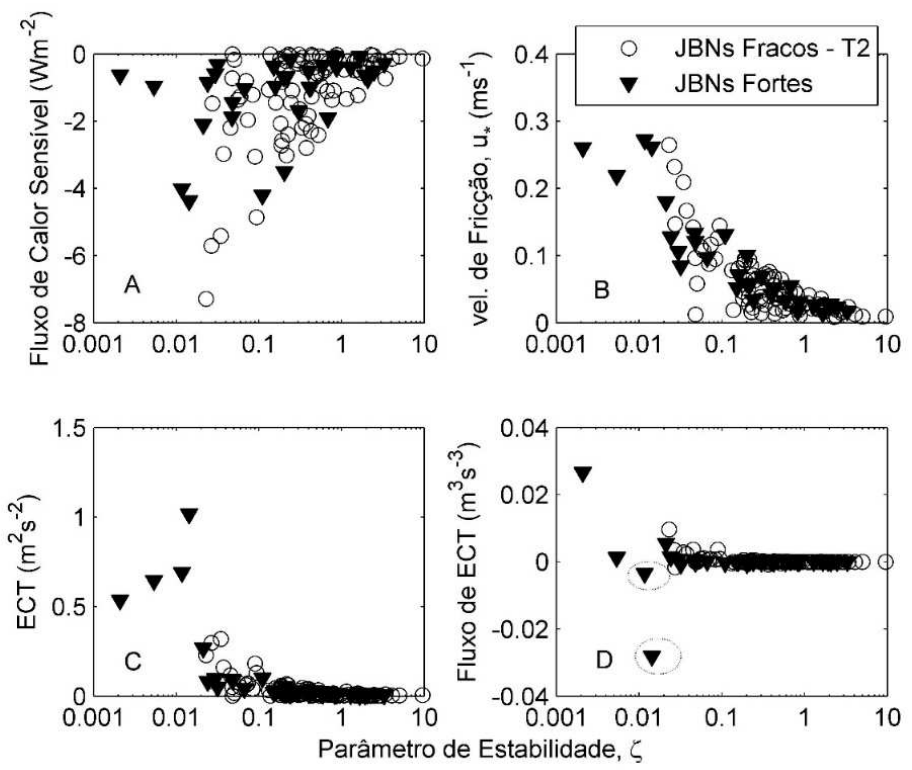

Figura 6 - Dispersão do parâmetro de estabilidade Monin-Obukhov em relação ao fluxo de calor sensível (A), velocidade de fricção (B), energia cinética turbulenta (C) e fluxo de energia cinética turbulenta (D), calculados durante eventos de JBNs fortes (triângulos pretos) e fracos-tipo 2 (círculos brancos). Os pontos dentro das elipses são correspondentes a um evento de jato forte que apresentou $Z_{\mathrm{j}}$ de $320 \mathrm{~m}$.

fortes. Este resultado, sugere que mesmo os JBNs fracos podem aumentar a turbulência atmosférica à superfície, desde que este aconteça em altura suficientemente baixa. Já em relação a estabilidade atmosférica, tanto para JBNs fortes quanto para JBNs fracos-tipo 2, na maioria dos casos os pontos estiveram dentro do regime intermediário. Por outro lado, cerca de $22 \%$ dos casos de JBNs fortes estiveram dentro de regime fracamente estável, enquanto que apenas aproximadamente 3\% dos casos de jatos fracos (tipo 1 e tipo 2) encontraram-se neste regime. É importante destacar ainda que nos casos de JBNs fracos tipo 1, o maior percentual dos pontos encontraram-se dentro do regime muito estável (aproximadamente 54\%).

Para tentar entender melhor como os JBNs podem afetar a estabilidade e a turbulência atmosférica à superfície, duas noites foram selecionadas para estudo de caso (Figura 7). A primeira, corresponde àquela em que o jato forte e mais baixo 

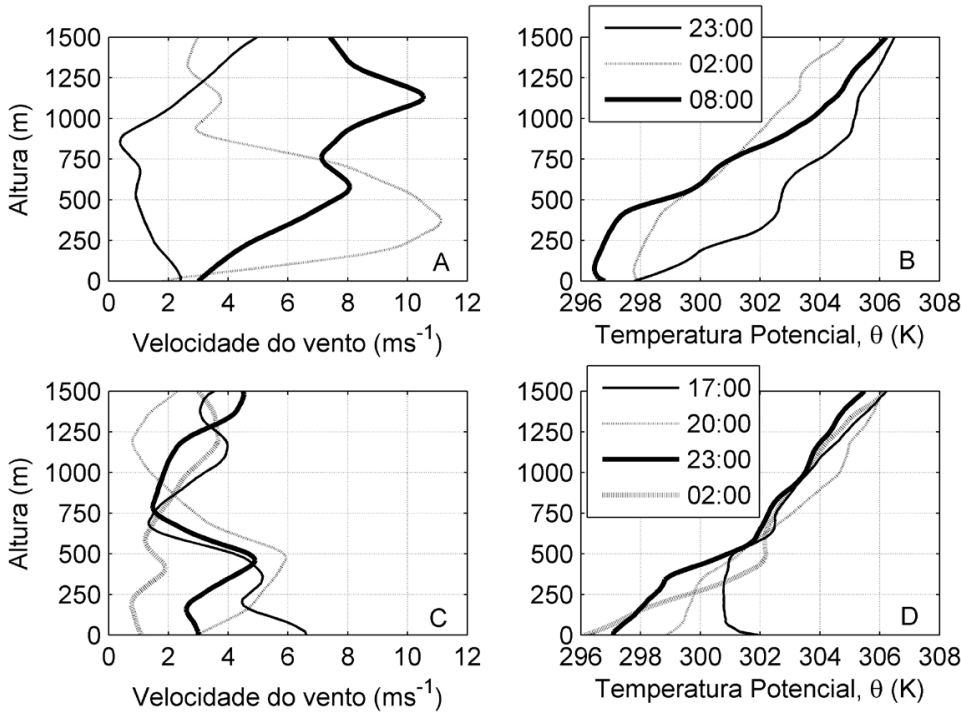

Figura 7 - Evolução do perfil vertical da velocidade do vento e da temperatura potencial para a noite 17-18 de fevereiro (A e B, respectivamente) e para a noite $04-05$ de fevereiro ( $\mathrm{C}$ e $\mathrm{D}$, respectivamente).

ocorreu e, ao mesmo tempo, os dados de superfície estavam completos (noite 17-18 de fevereiro, Figura 7A). Na outra, observa-se apenas perfis de velocidade do vento com JBNs fracos ou sem jato (noite 04-05 de fevereiro, Figura 7C). Esta última noite foi também escolhida por ser a que apresentou maior número de perfis de vento com a forma desejada para o estudo de caso.

Comparando a evolução temporal da temperatura do ar a $6 \mathrm{~m}$ de altura para as duas noites (Figura 8B), significantes diferenças são notadas. Tipicamente, a temperatura decai durante a noite, devido ao saldo de radiação negativo, todavia, a variação da temperatura entre $20 \mathrm{HL}$ e $06 \mathrm{HL}(\Delta \mathrm{T})$ é mais que o dobro maior, na noite com atividade de jato fraco $\left(\Delta \mathrm{T}=2,2^{\circ} \mathrm{C}\right)$ em relação àquela com jato forte $\left(\Delta \mathrm{T}=0,8^{\circ} \mathrm{C}\right)$. É importante destacar ainda, que a temperatura aumenta em certos períodos da noite 17-18 de fevereiro. Isso acontece provavelmente devido ao transporte de ar mais quente de camadas superiores da atmosfera para a superfície. Tal transporte gera uma camada bem misturada desde a superfície até cerca de $250 \mathrm{~m}$ de altura nesta noite às $2 \mathrm{HL}$ (Figura 7B). Outro fato a se comentar é que a injeção de turbulência não é contínua durante toda a noite, ela ocorre de forma intermitente, isso fica evidente no sinal da velocidade vertical do vento (Figura 8A).

Devido a turbulência aumentada durante a noite com atividade de jato forte, claras diferenças são verificadas na evolução temporal de $\mathrm{H}, \mathrm{u} *$ e ECT para as duas noites selecionadas (Figura $8 \mathrm{C}$ ). Os valores dessa variáveis são consideravelmente maiores, em certos períodos, durante a noite com atividade de jato forte do que durante a noite com atividade de JBNs fracos. Devido à baixa resolução temporal nos dados de radiossondagem atmosférica, não é possível determinar a hora exata em que o jato iniciou e cessou. Porém pode-se perceber que o jato das 02:00 HL (Figura 7A) coincide com o período em que a turbulência esteve elevada. Vale ressaltar que nesse horário aconteceu o segundo maior pico de ECT (Figura 8C)

Fitzjarrald e Moore (1990), estudando possíveis mecanismos que podem promover mistura turbulenta entre a floresta Amazônica e a atmosfera durante noite, encontraram significantes trocas mesmo em condições de céu claro, concluindo assim que outros mecanismos poderiam ser capazes de acoplar esses dois ambientes. Os resultados encontrados nesta pesquisa parecem corroborar tais afirmações, e sugerem que a presença JBNs, dependendo de sua velocidade e altura em que ocorre, pode influenciar tanto a estrutura da turbulência quanto a estabilidade à superfície.

$\mathrm{O}$ fato de que um jato forte, propagando-se em uma altitude suficientemente baixa, poder aumentar a turbulência à superfície, sugere que a teoria de blindagem por cisalhamento não se aplica ao sítio experimental estudado (Duarte et al., 2012). Uma vez que tal teoria prevê justamente o contrário, ou seja, a medida que o cisalhamento do vento aumenta, mais dificuldade um vórtice teria para se propagar de uma certa altitude para a superfície (Smedman et al., 2004; Prabha et al., 2008).

\section{CONCLUSÕES}

Neste trabalho JBNs foram identificados em $60 \%$ dos 235 perfis de vento analisados, dos quais $53 \%$ considerou-se fracos e $15 \%$ fortes. Os JBNs com $Z_{j}$ mais próximos da inversão 

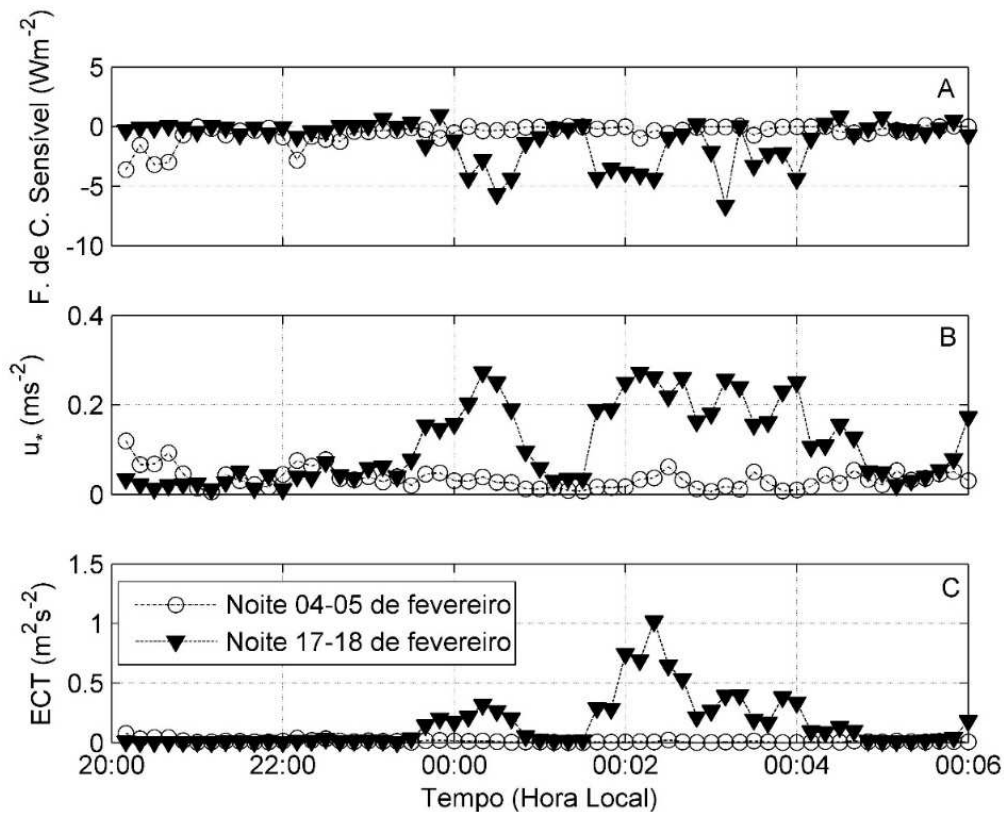

Figura 8 - Evolução temporal noturna do fluxo de calor sensível (A), velocidade de fricção (B) e energia cinética turbulenta (ECT) para a noite com atividade de jato forte (triângulos pretos) e para noite com atividade de jato fraco (circulos brancos).

térmica de superfície provavelmente aparecem devido ao desacoplamento friccional entre o escoamento atmosférico e a superfície, o chamado efeito de Blackadar. Por outro lado, efeitos de meso e grande escala, por si só, ou em conjunto com o efeito de Blackadar, podem ser responsáveis pelo o aparecimento de JBNs que se propagam em maiores altitudes.

Três regimes de estabilidade foram estabelecidos para a CLN da área de estudo: fracamente estável $(\zeta \leq 0,03)$; transição $(0,03<\zeta \leq 0,71)$ e muito estável $(\zeta>0,71)$. Foi observado que cerca de $22 \%$ dos casos de JBNs fortes estiveram dentro do regime fracamente estável, enquanto que apenas aproximadamente $3 \%$ dos casos de jatos fracos (tipo 1 e tipo 2 ) encontraram-se neste regime. Outro resultado interessante é que nos casos de JBNs fracos tipo 1, o maior percentual dos pontos encontraram-se dentro do regime muito estável (aproximadamente 54\%). Em relação a estrutura da turbulência, maiores valores médios de $\mathrm{u}_{*}$ e ECT foram observados durante a ocorrência de JBNs fortes do que durante eventos de JBNs fracos-tipo 2 (ocorrem entre a superfície até de $500 \mathrm{~m}$ de altura). Esta última classe de jato, por sua vez, apresentou maiores valores de $\mathrm{u}_{*}$ e ECT à superfície durante sua atuação do que durante eventos de JBNs fracos-tipo 1 (ocorrem acima de 500 $\mathrm{m}$ de altura).

Estes resultados sugerem que os JBNs com velocidades suficientemente altas e dependendo da altura em que ocorrem, podem aumentar a turbulência e introduzir fraca estabilidade atmosférica à superfície. Em casos bastante específicos de JBNs, pode estar presente uma camada limite invertida na área de estudo, em que a maior fonte de turbulência não está na interação do escoamento com a superfície, e sim gerada pelo próprio jato. Nossos resultados indicam também que a teoria de blindagem por cisalhamento não se aplica ao sítio experimental estudado.

As análises realizadas neste trabalho, a partir conjunto de dados utilizados, poderia ser enriquecida se o desenho experimental da coleta das observações fosse mais voltado para este tipo de estudo, ou seja, estudos em microescala, no âmbito da CLN. As limitações na disponibilidade de dados e a falta de uma melhor resolução na evolução temporal da velocidade do vento podem ter influenciado as análises e as conclusões alcançadas. Deste modo, medidas da evolução temporal da velocidade do vento realizadas por meio de SODAR, ou mesmo balão cativo com uma maior amostragem devem ser feitas em trabalhos futuros, visando aumentar o conhecimento de como os JBNs podem interferir nas trocar de massa, energia e momentum durante a noite na Amazônia.

\section{AGRADECIMENTOS}

Esta Pesquisa foi financiada por meio de bolsa de mestrado concedida pelo CNPq, processo $\mathrm{n}^{\circ} 133428 / 2011-7$, através do programa de Pós graduação em Clima e Ambiente INPA/UEA. Os autores agradecem ao Programa LBA pelos dados concedidos e a UEA pelo apoio logístico.

\section{REFERENCIAS}

ANDREAS, E. L.; CLAFFEY, K. J.; MAKSHTAS, A. 2000. Low-level atmospheric jets and inversions over the western 
Weddell Sea. Boundary-Layer Meteorology, 97: 459-486. ARYA, S. P. Introduction to micrometeorology. 2001. 2da ed. San Diego, California, 2001, 307p.

BANTA, R. M.; NEWSOM, R. K.; LUNDQUIST, J. K.; PICHUGINA, Y. L.; COULTER, R. L.; MAHRT, L. 2002. Nocturnal low-level jet characteristics over Kansas during CASES-99. Boundary- Layer Meteorology, 105: 221-252.

BANTA, R. M.; PICHUGINA, Y. L.; BREWER, W. A. 2006. Turbulent Velocity-Variance Profiles in the Stable Boundary Layer Generated by a Nocturnal Low-Level Jet. Journal of the Atmospheric Sciences, 63: 2700-2719.

BANTA, R.M; PICHUNGINA Y,L, NEWSOM R, K. 2003. Relationships between low-level jet properties and turbulence kinetic energy in the nocturnal stable boundary Layer. Journal of the Atmospheric Sciences, 60: 25492555.

BLACKADAR, A. K. 1957. Boundary Layer Wind Maxima and their Significance for the Growth of Nocturnal Inversions. Bulletin American Meteorological Society, 38: 283-290. COHEN, J. C. P.; SÁ, L. D. A.; NOGUEIRA, D. S.; GANDU, A. W. 2006. Jatos de baixos níveis acima da floresta amazônica em Caxiuanã. Revista brasileira de meteorologia, 21: 271-282.

DUARTE, H, F.; LECLERC, M. Y.; ZHANG, G. 2012. Assessing the shear-sheltering theory applied to low-level jets in the nocturnal stable boundary layer. Theoretical and Applied Climatology, 110: 359-371.

FISCH, G. 1995. Camada limite amazônica: aspectos observacionais e de modelagem. Tese de Doutorado, Instituto Nacional de Pesquisas Espaciais, São José dos Campos, São Paulo. 171p.

FITZJARRALD, D. R.; MOORE, K. E. 1990. Mechanisms of nocturnal exchange between the rain forest and the atmosphere. Journal of Geophysical Research, 95: 1683916850.

GRECO, S.; ULANSKY, S.; GARSTANG, M.; HOUSTON, S. 1992. Low level nocturnal wind maximum over the Central Amazonian Basin. Boundary-Layer Meteorology, 58: 91-115.

HUNT, J. C. R.; DURBIN, P. A. 1999. Perturbed vortical layers and shear sheltering. Fluid Dynamics Research, 24: 375-404.

KARIPOT, A.; LECLERC, M. Y.; ZHANG, G.; KEITH, F. L.; NAGY, J. J. H.; HENDREY, G. R..; STARR, G. 2008. Influence of nocturnal low-level jet on turbulence structure e $\mathrm{CO}_{2}$ flux measurements over a forest canopy. Journal of geophysical research, v113: 1-12.
LONGO, M.; ALBRECHT, R. I.; MACHADO, L. A. T.; FISCH, G.; SILVA DIAS, M. A. F. 2002. Controle de qualidade dos dados de radiossondagem da Campanha WET-AMC/LBA. Revista Brasileira de Meteorologia, 17: 243-253.

MAHRT, L. 1985. Vertical Structure and Turbulence in the Very Stable Boundary Layer. Journal of the Atmospheric Sciences, 42: 2333-2349.

MAHRT, L. 1999. Stratified atmospheric boundary layers. Boundary-Layer Meteorology, 90: 375-396.

MAHRT, L.; SUN, J.; BLUMEN, W.; DELANY, T.; Oncley, S. Nocturnal boundary-layer regimes. 1998. Boundary-Layer Meteorology, 88: 255-278.

NOGUEIRA, D, S. 2008. Aspectos observacionais e numéricos da interação floresta-atmosfera na Amazônia Oriental: fenômenos turbulentos noturnos, dissertação de mestrado, Universidade Federal do Pará/Museu Paraense Emílio Goeldi/Embrapa Amazônia Oriental, Belém, Pará. 141p.

OLIVEIRA, A. P.; FITZJARRALD, D. R. 1993. The Amazon River breeze and the local boundary layer I: observations. Boundary-Layer Meteorology, 63: 141-162.

PRABHA, T. V.; LECLERC, M. Y.; KARIPOT, A.; HOLLINGER, D. Y.; MURSCHRADLGRUBER, E. 2008. Influence of Nocturnal Low-level Jets on Eddy-covariance Fluxes over a Tall Forest Canopy. Boundary-Layer Meteorology, 126: 219-236.

SANTOS, R. M. N. 2005. Estudos da camada limite noturna na Amazônia. Tese de Doutorado, Instituto Nacional de Pesquisas Espaciais, São José dos Campos, São Paulo. 177 p.

SANTOS, R. M. N.; FISCH, G.; DOLMAN, A. J; WATERLOO, M. 2007. Modelagem da camada limite noturna (CLN) durante a época úmida na Amazônia, sob diferentes condições de desenvolvimento. Revista Brasileira de Meteorologia, 22: 387-407.

SMEDMAN, A. S.; HÖGSTRÖM, U.; HUNT, J. C. R. 2004. Effects of shear sheltering in a stable atmospheric boundary layer with strong shear. Quarterly Journal of the Royal Meteorological Society. 130: 31-50.

STULL, R. B. 1988. An introduction to boundary layer meteorology. Dordrecht: Kluwer Academic Publishers, 1988, 666p. 